\title{
Effets du pH et de la forme ionique sur l'affinité lysozyme-caséines
}

\author{
J.L. Thapon et G. BRUlé \\ I.N.R.A., Laboratoire de Recherches de Technologie laitière \\ 65, rue de Saint-Brieuc - 35042 Rennes cedex, France
}

\section{Résumé}

On a étudié l'influence de différents facteurs sur l'affinité du lysozyme pour la caséine entière, les caséines $\alpha_{\mathrm{S1}}, \beta$ et $\chi$.

L'affinité lysozyme-caséine diminue avec la baisse du pH. A pH 4,8, $100 \%$ du lysozyme ajouté (150 mg/g de caséine) demeure lié à la caséine entière et à la caséine $\alpha_{S 1}$. A ce même $\mathrm{pH}$, la caséine $\beta$ en a déjà relargué $80 \%$. La caséine $\chi$ se comportait différemment puisqu'elle ne fixait quasiment pas de lysozyme dans toute la gamme de $\mathrm{pH}$ étudiée (de 7 à 4,0).

On a également montré l'effet de la force ionique sur l'affinité lysozyme-caséine. Pour une concentration en chlorure de sodium de $4 \mathrm{~g} / 1,100 \%$ du lyzozyme rajouté sont fixés à la caséine $\alpha_{\text {s1 }}$. A cette concentration, la caséine entière et la caséine $\beta$ n'en retiennent plus que 60 et $10 \%$, respectivement. A la quantité maximale de chlorure de sodium rajoutée $(18,2 \mathrm{~g} / \mathrm{l})$ voisine de celle rencontrée dans la phase soluble du fromage, la caséine entière ne fixe plus que $21 \%$ du lysozyme rajouté.

On a enfin montré que la fixation du lyzozyme à la caséine entraînait une diminution de son activité d'un facteur voisin de 1000 .

Mots-clés : Lysozyme - Caséine - pH - Force ionique - Affinité.

\section{Introduction}

L'addition de lysozyme au lait de fabrication des pâtes pressées cuites est un des moyens utilisés aujourd'hui par l'industrie laitière pour lutter contre le développement des bactéries butyriques, et en particulier de Clostridium tyrobutyricum, provenant des ensilages de mauvaise qualité. Autorisé à la dose maximale de $30 \mathrm{mg} / \mathrm{l}$, le lysozyme limite ou retarde le développement des bactéries butyriques et permet un meilleur classement des fromages fabriqués. Cependant, 
l'efficacité de ce moyen de lutte contre la contamination des laits d'ensilage est encore souvent contestée, le coût élevé du lysozyme environ $1300 \mathrm{~F} / \mathrm{kg}$, soit $0,50 \mathrm{~F}$ en moyenne par kilo de fromage fabriqué - n'étant pas toujours compensé par le gain de qualité attendu. Le comportement du lysozyme ajouté au lait de fabrication fromagère est encore assez mal connu. GREEN et MARSHALL (1977) et PIERRE (1983) ont montré que l'addition de lysozyme au lait entraînait des modifications du processus de coagulation par la présure en raison d'une modification de la charge des micelles de caséines. En effet, le lysozyme, très chargé positivement au pH du lait $(\mathrm{pH}$ 6,7), se fixe très rapidement et très efficacement sur les caséines, entraînant une réduction de la charge globale des micelles.

Une des difficultés essentielles de l'étude du comportement du lysozyme en mélange avec les caséines réside dans le fait que la méthode de dosage de son activité biologique (SMOLELIS et Hartsell, 1949 ) est longue et fastidieuse et que le trouble apporté par les caséines en solution complique encore cette méthode de mesure spectrophotométrique.

A l'aide de la méthode de dosage du lysozyme par chromatographie liquide haute pression mise au point par THAPON et BRULÉ (1982), nous avons étudié le comportement de l'affinité du lysozyme vis-à-vis de la caséine entière, des caséines $\alpha_{s_{1}}, \beta$ et $\chi$, ainsi que l'influence du $\mathrm{pH}$ et de la force ionique sur cette affinité.

\section{Matériel}

\section{A. Lysozyme}

Le lysozyme de blanc d'œuf de poule utilisé était du chlorhydrate de lysozyme Afilact, préparation enzymatique fabriquée par CODIPI (12, rue Barbès, 92300 Levallois-Perret) ou du lysozyme pur cristallisé (Bacto-lysozyme DIFCO, réf. 0465) pour les études d'activité biologique.

\section{B. Caséine entière et caséines purifiées}

La caséine entière était obtenue à partir de lait préalablement écrémé, par précipitation avec $\mathrm{HCl} \mathrm{N}$ à $\mathrm{pH} 4,6$, lavage du précipité à l'eau distillée et remise en solution en ajustant le $\mathrm{pH}$ à 7 par addition de soude $\mathrm{N}$. Après reprécipitation, lavage et resolubilisation, la caséine était lyophilisée.

Les préparations de caséines purifiées ont été effectuées selon la méthode de ZitTle et Custer (1963) pour les caséines $\alpha_{\mathbb{S}_{1}}$ et $\chi$ et par la méthode de MERcier et al. (1968) pour la caséine $\beta$. Après 
précipitations successives et dialyse, les solutions étaient lyophilisées et conservées à $4^{\circ} \mathrm{C}$. La pureté des fractions a été contrôlée par chromatographie liquide haute pression.

\section{Chromatographie Liquide Haute Pression (CLHP)}

L'appareil utilisé était un chromatographe en phase liquide et à élution isocratique Waters équipé d'une colonne Micropack TSK $3000 \mathrm{SW}$ de $7,5 \mathrm{~mm}$ de diamètre et $30 \mathrm{~cm}$ de longueur et d'un passeur d'échantillons. On injectait $30 \mu \mathrm{l}$ de solution en tête de colonne. L'élution se faisait à température ambiante sous une pression de 15 atmosphères et un débit de $1 \mathrm{ml} / \mathrm{min}$. Le tampon d'élution utilisé était une solution de dihydrogénophosphate de sodium $0,01 \mathrm{M}$, ajustée à $\mathrm{pH}$ 2,2 par de l'acide orthophosphorique. Un intégrateur Waters Modèle 730 calculait automatiquement la surface des pics d'élution détectés à la sortie de la colonne par un détecteur UV muni d'un filtre à $280 \mathrm{~nm}$.

\section{Méthodes}

\section{A. Dosage du lysozyme}

En solution pure ou en mélange avec de la caséine entière ou différentes caséines purifiées (caséine $\alpha_{\mathbb{S}_{1}}, 6$ et $\chi$, le lysozyme était dosé quantitativement selon la méthode de THAPON et BRULÉ (1982) ; son activité biologique était déterminée par la méthode de SMolelis et Hartsell (1949).

\section{B. Effet du pH sur l'affinité lysozyme-caséines}

L'effet du pH sur l'affinité du lysozyme pour les caséines était évalué en dosant le lysozyme par CLHP après élimination des caséines.

A $30 \mathrm{ml}$ d'une solution de caséine entière à $4 \mathrm{mg} / \mathrm{ml}$ dans du chlorure de calcium $0,01 \mathrm{M}$, on ajoutait $1,2 \mathrm{ml}$ d'une solution de lysozyme à $5 \mathrm{mg} / \mathrm{ml}$ également dans du chlorure de calcium $0,01 \mathrm{M}$ (concentration du lysozyme dans le mélange : 0,19 $\mathrm{mg} / \mathrm{ml}$ ).

Sept fractions identiques étaient amenées à différents $\mathrm{pH}$ par addition de soude ou d'acide chlorhydrique et les volumes ajustés avec de l'eau distillée. Après avoir porté les échantillons au bainmarie à $40^{\circ} \mathrm{C}$, on ajoutait $130 \mu \mathrm{l}$ d'une dilution au $1 / 10^{\circ}$ d'une présure du commerce de force 10000 . Au bout d'un quart d'heure, les échantillons étaient filtrés sur papier Whatman $N^{\circ} 42$ et on dosait le 
lysozyme par CLHP dans le filtrat dilué deux fois avec le tampon phosphate $\mathrm{pH} 2.2$.

L'affinité du lysozyme pour les caséines purifiées (caséines $\alpha_{\mathrm{s}}$, $\beta$ et $\chi$ ) était déterminée selon le même principe. On mélangeait $25 \mathrm{ml}$ d'une solution de caséine $\alpha_{\mathrm{s}_{1}}, \beta$ ou $\chi$ à $1,33 \mathrm{mg} / \mathrm{ml}$ dans de l'eau distillée et $1 \mathrm{ml}$ d'une solution de lysozyme à $5 \mathrm{mg} / \mathrm{ml}$. Le $\mathrm{pH}$ était ensuite ajusté aux valeurs désirées. Pour éliminer les caséines, on précipitait les caséines $\alpha_{\mathbb{S}_{1}}$ et $\beta$ en ajoutant au mélange lysozyme-caséines $250 \mu \mathrm{l}$ d'une solution de chlorure de calcium $1 \mathrm{M}$ et en incubant $15 \mathrm{~min}$ à $40^{\circ} \mathrm{C}$. Pour la caséine $x$, la précipitation était réalisée par addition de $100 \mu \mathrm{l}$ de présure de force 1000 et de $250 \mu \mathrm{l}$ de la solution de chlorure de calcium molaire. Les échantillons étaient filtrés sur papier Whatman $\mathrm{N}^{\circ} 42$ et le lysozyme dosé par CLHP sur le filtrat dilué 2 fois dans le tampon phosphate $\mathrm{pH} 2,2$. L'élimination totale des caséines a pu être vérifiée par l'absence des pics d'élution correspondants dans les diagrammes d'élution en CHLP.

Les différences d'affinité du lysozyme pour les caséines $\alpha_{\mathbb{S}_{1}}$ et $\beta$ ont été déterminées à $\mathrm{pH} 6,6$. A $3 \mathrm{ml}$ de solution de caséine $\alpha_{\mathrm{st}}$ ou $\beta$ on ajoutait $1 \mathrm{ml}$ d'une solution de lysozyme de façon à ce que sa concentration dans le mélange varie de 0,1 à $2 \mathrm{mg} / \mathrm{ml}$. Les caséines étaient précipitées par addition de $50 \mu \mathrm{l}$ d'une solution de chlorure de calcium $1 \mathrm{M}$ et en incubant le mélange $15 \mathrm{~min}$ à $40^{\circ} \mathrm{C}$. Après centrifugation pendant 5 min à $500 \mathrm{~g}$, le lysozyme était dosé par CLHP sur le surnageant dilué deux fois dans le tampon phosphate.

\section{Effet de la force ionique sur l'affinité tysozyme-caséines}

Pour mesurer l'effet de la force ionique sur l'affinité lysozymecaséines, on mélangeait $3 \mathrm{ml}$ de solution de caséine entière, $\alpha_{\mathrm{s}_{1}}$ ou $\beta$ à $1,77 \mathrm{mg} / \mathrm{ml}$ et $1 \mathrm{ml}$ d'une solution de lysozyme à $0,8 \mathrm{mg} / \mathrm{ml}$, le mélange étant à $\mathrm{pH}$ 6,6. Du chlorure de sodium était alors ajouté de telle sorte que sa concentration dans le mélange lysozyme-caséines varie de 0,45 à $18 \mathrm{~g} / 1$. Les caséines étaient ensuite précipitées par addition de $40 \mu l$ d'une solution de chlorure de calcium $1 \mathrm{M}$, le précipité éliminé par centrifugation et le lysozyme dosé par CLHP sur le surnageant dilué 2 fois dans le tampon phosphate.

\section{Résultats}

\section{A. Séparation des caséines par CLHP}

Nous avons réalisé des solutions pures de caséines $\alpha_{\$_{1}}, \beta$ et $\chi$ aux concentrations respectives de $0,66 \mathrm{mg} / 1,0,66 \mathrm{mg} / \mathrm{ml}$ et $0,33 \mathrm{mg} / \mathrm{ml}$ dans du tampon phosphate $0,01 \mathrm{M}, \mathrm{pH} 2,2$. Après élution sur la 


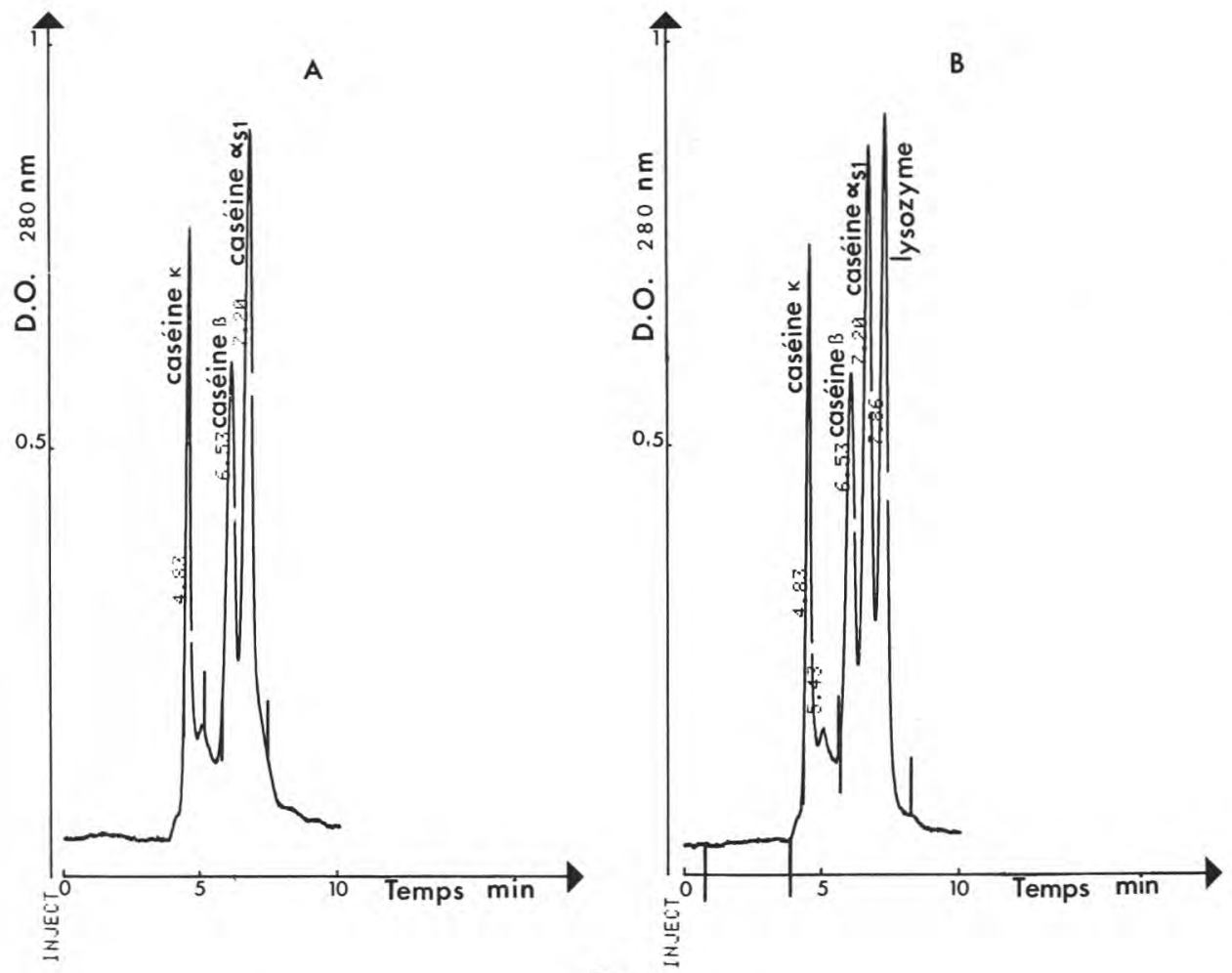

FIG. 1

Chromatogramme (CLHP) d'une solution pure de caséinate et de caséinate additionné de lysozyme

A : Solution pure de caséinate $(1,33 \mathrm{mg} / \mathrm{ml})$ dans un tampon phosphate, $p H$ 2,2.

$B$ : Mélange de caséinate $(1,33 \mathrm{mg} / \mathrm{ml})$ et de lysozyme $(0,1 \mathrm{mg} / \mathrm{ml})$ dans un tampon phosphate, $\mathrm{pH} 2,2$.

Chromatogram (HPLC) of a pure caseinate solution and of a blend of caseinate and lysozyme

A : Pure caseinate solution $(1.33 \mathrm{mg} / \mathrm{ml})$ in phosphate buffer $0.01 \mathrm{~N}-\mathrm{pH} 2.2$.

$B$ : Mix of caseinate $(1.33 \mathrm{mg} / \mathrm{ml})$ and lysozyme $(0.1 \mathrm{mg} / \mathrm{ml})$ in phosphate buffer $0.01 \mathrm{~N}-\mathrm{pH} 2.2$.

colonne, les temps de rétention observés étaient de 7,23 $\mathrm{min}, 6,56 \mathrm{~min}$ et 4,82 min. Nous avons ensuite analysé une solution dans le même tampon de caséine entière à la concentration de $1,33 \mathrm{mg} / \mathrm{ml}$. Le chromatogramme obtenu est donné dans la figure 1. Les temps de rétention obtenus pour les 3 principaux pics correspondant aux caséines $\alpha_{\mathrm{s}_{1}}, \beta$ et $\chi$ étaient respectivement de 7,23 $\mathrm{min}, 6,53 \mathrm{~min}$ et $4,86 \mathrm{~min}$, donc identiques à ceux obtenus avec les solutions pures de caséines. La concentration en caséine entière calculée à partir des surfaces des pics de caséines pures, c'est-à-dire considérées comme étalons, était de $1,26 \mathrm{mg} / \mathrm{ml}$, soit une différence de $4,8 \%$ avec la concentration réelle. 
L'addition de lysozyme à la caséine entière a permis de montrer que, dans les conditions d'analyse, il y avait une légère interférence entre le pic du lysozyme et le pic de caséine $\alpha_{s_{1}}$ (fig. 1). Cependant, le temps de rétention du lysozyme demeure le même lorsqu'il se trouve en solution pure ou en mélange avec les caséines (7,90 min).

\section{B. Influence du pH sur l'affinité lysozyme-caséines}

Les solutions de caséines $\alpha_{\mathrm{s}_{1}}, \beta$ et $\chi$ à $1,33 \mathrm{mg} / \mathrm{ml}$ dans l'eau distillée étaient limpides. Au cours de l'acidification, un trouble apparaissait à partir de $\mathrm{pH} 5,5$ et un début de précipitation à partir de $\mathrm{pH} 5,0$. L'addition de lysozyme $(0,19 \mathrm{mg} / \mathrm{ml}$ dans le mélange lysozyme-caséine) entraînait par contre l'apparition d'un trouble du mélange dès les $\mathrm{pH}$ voisins de 6,0 . La solution de caséine entière à $4,0 \mathrm{mg} / \mathrm{ml}$ était opalescente, même sans addition de lysozyme, dans toute la gamme de $\mathrm{pH}$ étudiée.

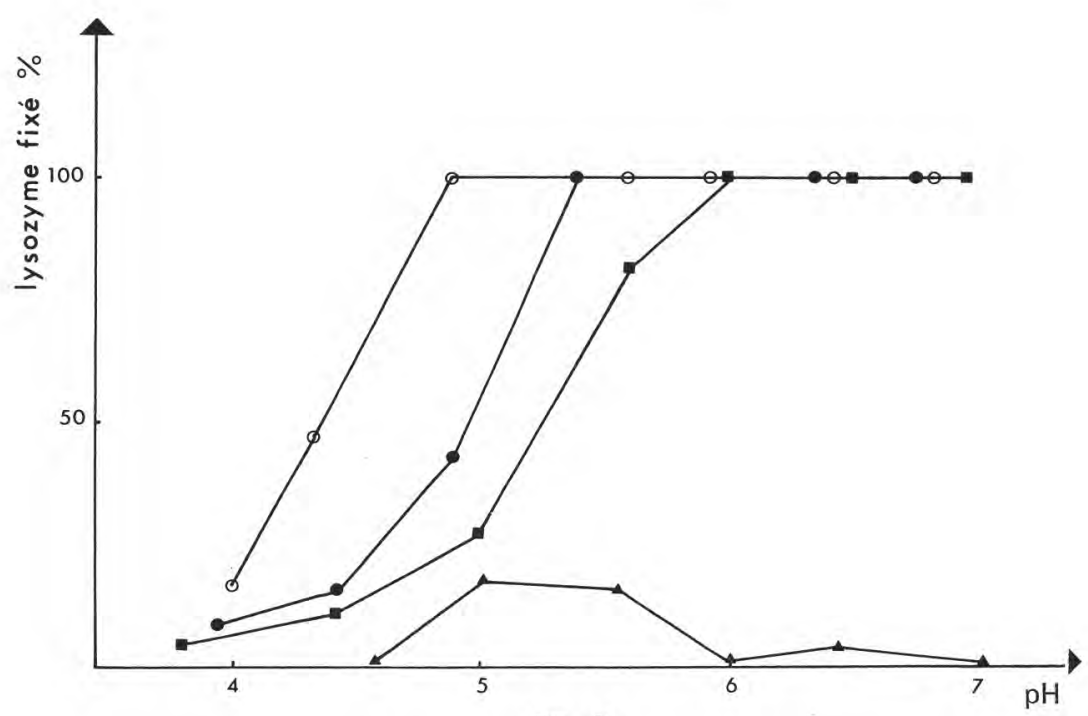

FIG. 2

Influence du pH sur l'affinité lysozyme-caséine

- caséine entière (caséine/lysozyme:21).

- caséine $\alpha_{\mathrm{s} 1} \quad$ (caséine/lysozyme: 7).

- caséine $\beta$ ( $"$ " $)$.

$\Delta$ caséine $\chi \quad(» \quad »)$.

Influence of $p H$ on lysozyme-casein affinity

- whole casein (casein/lysozyme:21).

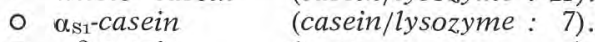

- $\beta$-casein ( " " $"$ ).

- $\chi$-casein ( $\gg »)$. 
La diminution du pH entrainnait une diminution de l'affinité du lysozyme pour la caséine entière et les caséines $\alpha_{\mathrm{s}_{1}}$ et $\beta$. La figure 2 montre que le lysozyme s'associe de façon préférentielle avec la caséine $\alpha_{s_{1}}$ puisqu'il faut descendre en dessous de $\mathrm{pH}$ 4,85 pour commencer à observer une diminution de cette affinité. A ce même $\mathrm{pH}$, le lysozyme a une affinité plus de 2 fois plus faible pour la caséine entière ( $42 \%$ de lysozyme fixé à $\mathrm{pH} 4,8)$. A pH 4,95, $26 \%$ du lysozyme se retrouve fixé à la caséine $\beta$. Le comportement de la caséine $\chi$ vis-à-vis du lysoyzme est différent de celui de la caséine entière et des caséines $\alpha_{\mathrm{s}_{1}}$ et $\beta$ puisqu'à tous $\mathrm{pH}$, cette molécule n'a pas ou très peu d'affinité pour le lysozyme. Seulement $15 \%$ du lysozyme se retrouve fixé à la caséine $x$ aux environs de $\mathrm{pH} 5,0$.

\section{Différence d'affinité du lysozyme vis-à-vis des caséines $\alpha_{\mathbb{s}_{1}}$ et $\beta$}

En accord avec la " théorie des équilibres multiples ", la liaison réversible d'un ligand et d'une macromolécule peut s'exprimer selon

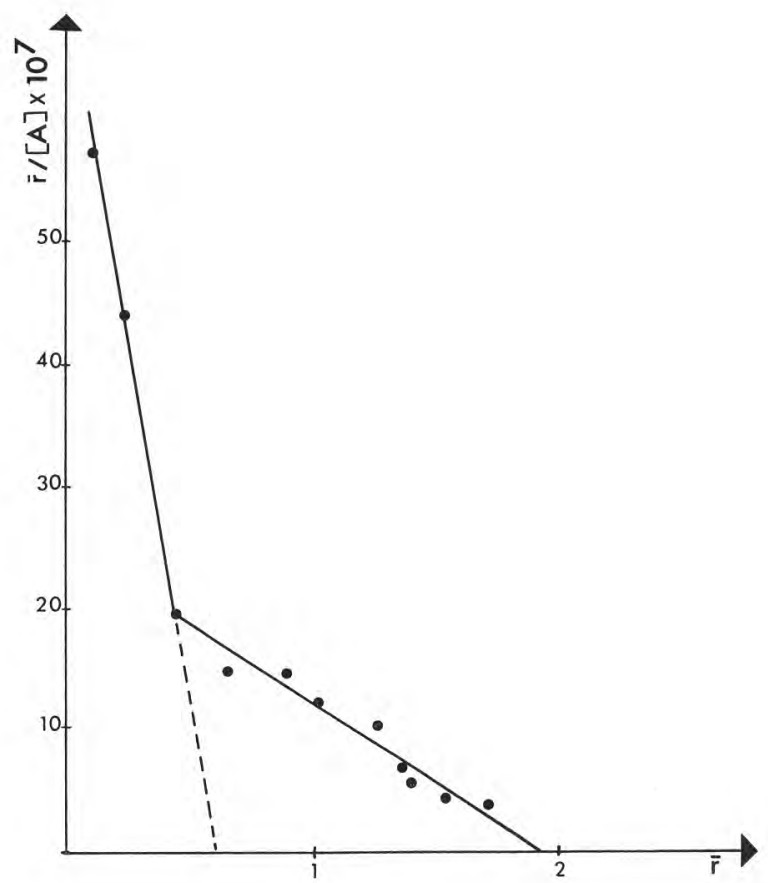

FIg. 3

Affinité du lysozyme pour la caséine $\alpha_{\mathrm{S} 1}$ (représentation de Scatchard).

Affinity between lysozyme and $\alpha_{\mathrm{S} 1}$-casein (Scatchard's representation). 
la formule suivante (KLOTZ, 1946; Scatchard, 1949; Wood et COOPER, 1970) :

$$
\overline{\mathrm{r}}=\sum_{\mathrm{i}}^{\mathrm{m}} \frac{\mathrm{n}_{\mathrm{i}} \mathrm{K}_{\mathrm{i}}[\mathrm{A}]}{1+\mathrm{K}_{\mathrm{i}}[\mathrm{A}]}
$$

$\overline{\mathrm{r}} \quad=$ nombre moyen de moles de ligand (lysozyme) associé par mole de macromolécule (caséine).

$\mathrm{m}=$ nombre de classes de sites de liaison indépendants.

$\mathrm{n}_{\mathrm{i}}$. nombre de sites pour chaque classe ..

$\mathrm{K}_{\mathrm{i}}=$ constante d'affinité des sites $\mathrm{n}_{\mathrm{i}}$.

$[\mathrm{A}]=$ concentration en ligand (lisozyme) à l'état libre.

L'affinité des caséines $\alpha_{\mathrm{s}_{1}}$ et $\beta$ à $\mathrm{pH}$ 6,6 vis-à-vis du lysozyme était déterminée à l'aide de la représentation de ScatchaRd (1949) où l'on représente $\bar{r} /[\mathrm{A}]$ en fonction de $\overline{\mathrm{r}}$ fig. 3 et 4 ). L'affinité de la caséine $\alpha_{s_{1}}$ vis-à-vis du lysozyme $\left(K_{i}=9,0 \times 10^{4}\right)$ est plus forte que celle de la caséine $\beta\left(\mathrm{K}_{\mathrm{i}}=6,8 \times 10^{4}\right)$. A pH 6,6, la caséine $\alpha_{\mathrm{s} 1}$ fixe 0,62 mole de lysozyme contre 0,43 mole pour la caséine $\beta$ soit une différence d'affinité de $30 \%$ entre les 2 caséines.

D. Effet de la force ionique sur l'affinité lysozyme-caséines

Nous avons examiné l'influence de la concentration en chlorure de sodium sur l'affinité du lysozyme pour les caséines. Les résultats

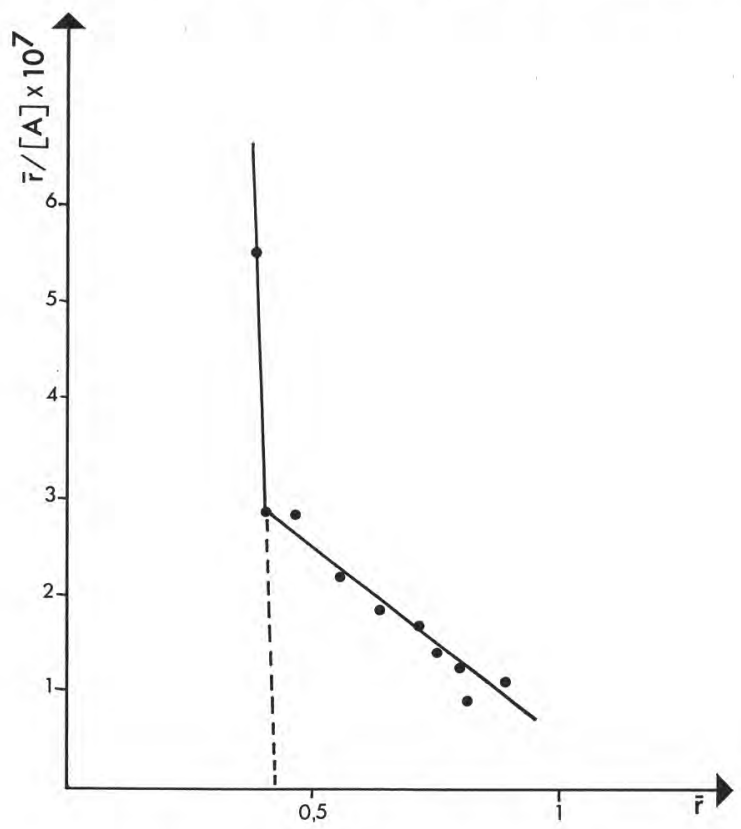

FIG. 4

Affinité du lysozyme pour la caséine $\beta$ (représentation de Scatchard). Affinity between lysozyme and $\beta$-casein (Scatchard's representation). 


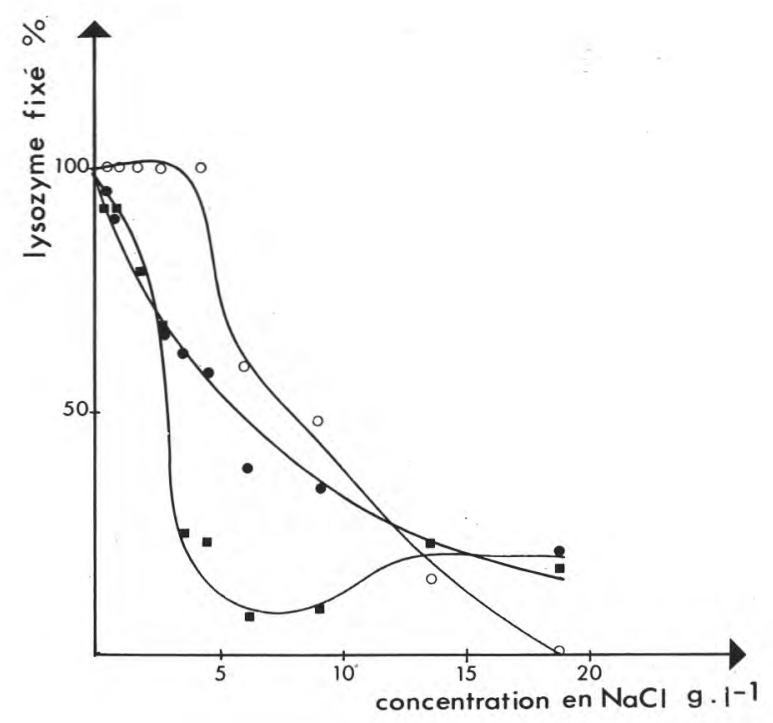

FIG. 5

Influence de la force ionique sur l'affinité lysozyme-caséine (caséine/lysozyme $=8,85$ )

$\circ$ caséine $\alpha_{\mathrm{s} 1}$.

- caséine $\beta$.

- caséine entière.

Influence of ionic strength on the affinity between lysozyme and caseins (casein/lysozyme : 8.85)

O $\alpha_{\mathbf{s}_{1}}$ casein.

- B-casein.

- whole casein.

obtenus en ajoutant à un mélange lysozyme-caséines des concentrations en chlorure de sodium allant de 0 à $18 \mathrm{~g} / 1$, sont réunis daris la figure 5 .

L'association lysozyme-caséines dépend étroitement de la force ionique du milieu. A pH 6,6 et sans chlorure de sodium, $100 \% \mathrm{du}$ lysozyme rajouté sont fixés par la caséine entière, la caséine $\alpha_{s_{1}}$ et la caséine $\beta(0,15 \mathrm{~g}$ de lysozyme par g de protéine). Pour une concentration en chlorure de sodium de $4 \mathrm{~g} / 1,100 \%$ du lysozyme sont fixés sur la caséine $\alpha_{\mathbb{S}_{1}}$, alors que la caséine entière et la caséine $\beta$ n'en retiennent respectivement plus que 60 et $10 \%$. A une concentration en chlorure de sodium voisine de celle rencontrée dans la phase soluble du fromage $(18,2 \mathrm{~g} / 1)$, la caséine entière ne fixe plus que $21 \%$ de la quantité du lysozyme rajouté. 


\section{E. Effet de l'affinité lysozyme-caséines sur son activité biologique}

Nous avons mesuré l'effet de l'affinité lysozyme-caséines sur son activité biologique à $\mathrm{pH}$ 6,2. A une solution pure de lysozyme de concentration $0,53 \mathrm{mg} / \mathrm{ml}$ déterminée par la méthode de SMoLELIS et HARTSELL, on ajoutait une solution de caséine entière de façon à avoir dans le mélange un rapport lysozyme caséine de $1-4$. L'activité biologique du lysozyme déterminée par la même méthode donnait alors une concentration en enzyme de $0,70 \mu \mathrm{g} / \mathrm{ml}$. Dans ces conditions, la totalité du lysozyme était liée aux caséines.

\section{Discussion}

Nous avons montrè que l'affinité du Iysozyme pour les caséines dépendait à la fois du $\mathrm{pH}$, de la force ionique et du type de caséine (caséine entière, caséines $\alpha_{S 1}, \beta$ et $\chi$ ). En ce qui concerne l'influence du $\mathrm{pH}$, le comportement des caséines entières, $\alpha_{\mathrm{s}_{1}}$ et $\beta$ est identique : leur affinité pour le lysozyme diminue avec l'abaissement du $\mathrm{pH}$. Cette affinité est la plus forte pour la caséine $\alpha_{\mathrm{s}_{1}}$ et, à $\mathrm{pH} 4,8,100 \%$ du lysozyme rajouté (150 $\mathrm{mg} / \mathrm{g}$ de caséine) demeurent liés à celle-ci. A pH 5,6 et à la même concentration lysozyme-caséine, la caséine $\beta$ a déjà relargué $81 \%$ du lysozyme rajouté. La caséine $\chi$ se comportait différemment puisqu'elle ne fixait pas ou peu de lysozyme à tous les pH étudiés. Une étude analogue du comportement de la caséine $\alpha_{\text {s } 2}$ devrait être entreprise.

Cette différence d'affinité des caséines pour le lysozyme peut être reliée à leur taux de phosphorylation, comme l'a confirmé la représentation de Scatchard. En effet, le taux de phosphorylation de la caséine $\alpha_{s_{1}}$ ( $8 \mathrm{P}$ par molécule) est plus élevé que celui de la caséine $\beta$ ( $5 \mathrm{P}$ par molécule), la caséine $\chi$ étant la moins phosphorylée parmi les caséines ( $1 \mathrm{P}$ par molécule). Des liaisons de nature électrostatique s'établiraient donc entre les groupements phosphates ionisés des caséines et les groupements $\varepsilon-\mathrm{NH}^{+}{ }_{3}$ des lysines du lysozyme. BRULÉ et FAUQUANT (1982) avaient trouvé des résultats analogues en étudiant le pouvoir chélatant des caséines $\alpha_{\mathbf{s}_{1}}$ et $\beta$ vis-à-vis des oligoéléments. Le comportement du lysozyme vis-à-vis des caséines confirme donc ce résultat. Quant à la diminution de l'aftinité des caséines $\alpha_{s_{1}}$ et $\beta$ pour le lysozyme parallèlement à la diminution du $\mathrm{pH}$, elle renforce l'hypothèse de la création de liaisons électrostatiques entre ces 2 types de protéines, les groupements phosphate des caséines subissant une régression d'ionisation au fur et à mesure que le $\mathrm{pH}$ se déplace vers les $\mathrm{pH}$ acides.

L'affinité du lysozyme pour les caséines $\alpha_{s_{1}}$ et $\beta$ se traduisait macroscopiquement par une augmentation du trouble du mélange qui pouvait s'expliquer par une diminution de la charge globale 
négative des molécules et un début d'agrégation de celles-ci. Cette modification de charge avait déjà été notée par GrEeN (1973), GREEN et MARSHALl (1977) et PIERRE (1983) étudiant l'influence de la charge de la micelle sur le phénomène de coagulation par la présure.

L'addition de quantités croissantes de chlorure de sodium au mélange lysozyme-caséine a permis de préciser l'influence de la force ionique sur l'affinité de ces deux protéines. L'addition de chlorure de sodium relargue le lysozyme fixé aux caséines. Ce relargage est plus important pour la caséine $\beta$ que pour la caséine $\alpha_{\mathbb{S}_{1}}$, la caséine entière ayant un comportement intermédiaire. Ceci confirme encore le rôle des groupements phosphate des caséines dans l'établissement des liaisons avec le lysozyme. La caséine la plus riche en phosphore est la moins sensible à l'addition de chlorure de sodium quant à la libération du Iysozyme qui lui est associé. En solution de force ionique voisine de celle du lait $(\mu=0,08$, équivalent environ à $5 \mathrm{~g} / 1$ de $\mathrm{NaCl}$ ), $60 \%$ du lysozyme ajouté à la concentration de $150 \mathrm{mg} / \mathrm{g}$ de caséine sont associés à la caséine entière. Dans la pratique industrielle, pour la fabrication des pâtes pressées cuites, et afin de lutter contre le développement de Clostridium tyrobutyricum, on ajoute du lysozyme au lait de fabrication à la dose de $25 \mathrm{mg}$ par litre, soit environ $1 \mathrm{mg}$ par $\mathrm{g}$ de caséine. Dans ces conditions, $60 \% \mathrm{du}$ lysozyme au minimum se trouvent à l'état complexé aux caséines et des liaisons avec les globules gras dépendant des mêmes facteurs ne sont pas à exclure. De plus, nous avons montré que son activitě biologique dans ces conditions diminuait environ d'un facteur 1000 . Au cours de la première étape de la fabrication du fromage, le développement de la fermentation lactique entraîne une acidification du lait. Pour les pâtes pressées cuites, le chauffage du caillé bloque l'acidification à $\mathrm{pH} \mathrm{5,2}$ par élimination de lactose et augmentation de l'extrait sec donc du pouvoir tampon des protéines. La totalité du lysozyme rajouté au lait demeurerait donc lié aux caséines et son activité biologique ne pourrait alors s'exprimer. Après salage du fromage, la concentration en chlorure de sodium dans la phase soluble atteignant $2 \%$, une partie du lysozyme serait alors relarguée et son activité biologique favorisée par une remontée du $\mathrm{pH}$. Ces hypothèses devront être vérifiées en faisant des dosages de lysozyme et des mesures de son activité biologique à différentes étapes de la fabrication du fromage.

\section{Remerciements}

Merci à Patrice MARTIN pour la préparation des caséines purifiées, à Alice PIERRE et Jean-Louis MAUBOIS pour la lecture critique du manuscrit. Merci également à Gabrielle pour le graphisme, à Pascaline pour la traduction et à Anne-Marie et Christiane pour la frappe. 


\section{Summary}

\section{Effect of $\mathrm{pH}$ and ionic strengh on lysozyme-caseins affinity}

The affinity of lysozyme for the $\alpha_{\mathrm{S}^{-}}, \beta-, \mathrm{K}-$ and whole caseins has been measured in different experimental conditions.

No interaction was observed between $\mathrm{K}$-casein and lysozyme in all the conditions tested $(\mathrm{pH} 7.0$ to $4.8, \mathrm{NaCl}: 4 \mathrm{~g} / 1)$. On the opposite, all the lysozyme is fixed on $\alpha_{\mathrm{s}^{-}}$casein whatever the $\mathrm{pH}$ is between 7.0 and 4.8 , or in presence of sodium chloride $(4 \mathrm{~g} / 1)$. The $\beta$-casein presents an intermediary affinitv - if $100 \%$ of lysozyme is fixed on it at $\mathrm{pH} 7.0$ only $20 \%$ remains at $\mathrm{pH} 4.8$. After adding $\mathrm{NaCl}(4 \mathrm{~g} / \mathrm{l})$, there is also a release of lysozyme, only $10 \%$ remaining on $\beta$-casein.

When whole casein is used, all the lysozyme is fixed from $\mathrm{pH} 7.0$ to 4.8 , but only $60 \%$ if $4 \mathrm{~g} / 1$ of $\mathrm{NaCl}$ is added. The influence of $\mathrm{NaCl}$ has also been tested at concentrations close to the highest encountered in cheese technology. In these case, only $21 \%$ of the lysozyme remains fixed.

Finally, we have shown that the interaction of lysozyme with casein brings about a decrease of activity by a factor 1000 .

Key words : Lysozyme - Casein - pH - Ionic strength - Affinity.

\section{Références bibliographiques}

Brulé G., Fauquant J., 1982. Interaction des protéines du lait et des oligoéléments. Lait, 62, 323-331.

GREEN M.L., 1973. Studies on the mechanisms of clotting of milk, Neth. Milk Dairy J., 27, 278-285.

Green M.L., Marshall R.J., 1977. The acceleration by cationic materials of the coagulation of casein micelles by rennet. J. Dairy Res, 44, 521-531.

KLOTZ I.M., 1946. The application of the law of mass action to binding by proteins. Interactions with calcium. Arch. Biochem., 9, 109-117.

Mercier J.C., Maubois J.L., Poznanski S., Ribadeau Dumas B., 1968. Fractionnement préparatif des caséines de vache et de brebis par chromatographie sur D.E.A.E. cellulose en milieu urée et 2 mercapto-éthanol. Bull. Soc. Chim. Biol., 50, 521-530.

PIERRE A., 1983. Influence de la modification de la charge des micelles de caséines sur le taux de caséinomacropeptide libéré par la présure au moment de la coagulation du lait. Lait, 63, 217-229.

SCATchaRd G.F., 1949. The attractions of proteins for small molecules and ions. Ann. N.Y. Acad. Sci., 51, 660-672.

SMolelis A.N., HaRtSell S.E., 1949. The determination of lysozyme. J. Bacteriol., $58,731-736$.

Thapon J.L., Brulé G., 1982. Dosage du lysozyme dans le blanc d'œuf par chromatographie liquide haute performance. Sci. Aliments, 2, 251-260.

Wood G.C., COOPER P.F. 1970. The application of gel filtration to the study of protein-binding of small molecules. Chromatograph. Rev., 12, 88-107.

Zittle C.A., Custer J.H., 1963. Purification and some of the properties of $\alpha_{\mathrm{s}}$-casein and $\chi$-casein. J. Dairy Sci., 46, 1183-1188. 